\title{
Dynamic fluorescent imaging with the activatable probe, Y-glutamyl hydroxymethyl rhodamine green in the detection of peritoneal cancer metastases: Overcoming the problem of dilution when using a sprayable optical probe
}

\author{
Yuko Nakamura ${ }^{1}$, Toshiko Harada ${ }^{1}$, Tadanobu Nagaya ${ }^{1}$, Kazuhide Sato ${ }^{1}$, Shuhei \\ Okuyama $^{1}$, Peter L. Choyke ${ }^{1}$, Hisataka Kobayashi ${ }^{1}$ \\ ${ }^{1}$ Molecular Imaging Program, Center for Cancer Research, National Cancer Institute, Bethesda, MD 20892, USA \\ Correspondence to: Hisataka Kobayashi, email: kobayash@mail.nih.gov
}

Keywords: kinetic map, green emitting probe, autofluorescence, $\gamma$-glutamyltranspeptidase, peritoneal cancer metastases

Received: April 25, $2016 \quad$ Accepted: May 22, $2016 \quad$ Published: June 07, 2016

\section{ABSTRACT}

Optical fluorescence-guided imaging is increasingly used to guide surgery and endoscopic procedures. Activatable probes are particularly useful because of high target-to-background ratios that increase sensitivity for tiny cancer foci. However, green fluorescent activatable probes suffer from interference from autofluorescence found in biological tissue. The purpose of this study was to determine if dynamic imaging can be used to differentiate specific fluorescence arising from an activated probe in a tumor from autofluorescence in background tissues especially when low concentrations of the dye are applied. Serial fluorescence imaging was performed using various concentrations of y-glutamyl hydroxymethyl rhodamine green (gGluHMRG) which was sprayed on the peritoneal surface with tiny implants of SHIN3DsRed ovarian cancer tumors. Temporal differences in signal between specific green fluorescence in cancer foci and non-specific autofluorescence in background tissue were measured at 5, 10, 20 and 30 min after application of gGlu-HMRG and were processed into three kinetic maps reflecting maximum fluorescence signal (MF), washin rate (WIR), and area under the curve (AUC), respectively. Using concentrations up to $10 \mu \mathrm{M}$ of gGlu-HMRG, the fluorescence intensity of cancer foci was significantly higher than that of small intestine but only at $\mathbf{3 0}$ min. However, on kinetic maps derived from dynamic fluorescence imaging, the signal of cancer foci was significantly higher than that of small intestine after only $\mathbf{5}$ min even at concentrations as low as $2.5 \mu \mathrm{M}$ of gGlu-HMRG $(p<0.01)$. At lower concentrations, kinetic maps derived from dynamic fluorescence imaging were superior to unprocessed images for cancer detection.

\section{INTRODUCTION}

In many oncologic procedures, the ability to completely resect tumors is important for long term durable responses. Although large tumors are visible to the unaided human eye and can be readily removed, tiny foci ( 2 to $3 \mathrm{~mm}$ ) of cancer metastases or invading cells may be more difficult to see. Consequently, optical fluorescence-guided imaging is increasingly used as an aid to surgery or endoscopy to guide the detection of tiny tumor foci. Optical fluorescence imaging offers high sensitivity, low cost, portability, realtime capabilities, and importantly, absence of ionizing radiation [1-5] and thus, there is much interest in this topic.
There are two major categories of fluorescent probes that have been used in this context: 'always on' and activatable probes [6]. Always-on probes fluoresce regardless of whether they are bound to the target tissue and thus, have the disadvantage of high background signal. One approach is to wait for clearance of background signal so that adequate target-to-background ratios (TBRs) can be achieved but signal within the tumor also decreases, lowering sensitivity. On the other hand, activatable probes only become fluorescent after they come in contact with the target tissue. Thus, this class of optical probes have lower background signals, but require rapid activation to be practical in the clinical environment [7]. One 
common approach for activating optical probes is to make use of specific enzymatic activity found in the tumor microenvironment but not in normal tissues [8].

$\gamma$-glutamyl hydroxymethyl rhodamine green (gGluHMRG) is an activatable optical probe that produces the green fluorescent product, HMRG, after exposure to $\gamma$-glutamyltranspeptidase (GGT), a cell surface-associated (or bound) enzyme involved in cellular glutathione homeostasis. GGT is overexpressed in several human tumors, including cervical and ovarian cancers [9-13]. gGlu-HMRG has been reported to be able to detect intraperitoneal metastases in preclinical mouse models within $10 \mathrm{~min}$ of topical application because of its rapid and strong activation upon contact with GGT [9]. This probe is also sprayable onto the surface of tumors, making it quite convenient to deliver to an entire surgical or endoscopic field. Several studies have assessed the diagnostic performance of gGlu-HMRG for various cancers and more studies are underway [14-17].

The choice of emission wavelength for an optical probe depends on use for which it is intended. For lesions that might be hiding beneath the organ surface, near infrared light is preferred because of its depth of penetration in tissue. However, for surgical or endoscopic procedures, where the task is to detect surface lesions, shorter wavelengths can be considered. Green light is of interest because the human eye is exquisitely sensitive to it and it requires no special equipment for detection. However, green light also poses some challenges, particularly, interference from autofluorescence. In the case of detecting intraperitoneal metastases, autofluorescence of surrounding normal tissue, such as the small bowel, may hamper the detection of intraperitoneal lesions. This is especially true if the probe becomes diluted in pools of fluid in the body cavity and thus, emits lower intensity of light. One approach to reducing autofluorescence is to unmix known autofluorescence spectra from the optical probe spectra. However, current spectral imaging is time consuming requiring at least several seconds per frame and therefore, is not amenable to real time imaging during surgical or endoscopic procedures $[6,18]$.

One difference between the green light from an exogenous activatable fluorophore and endogenous autofluorescence is that the former is dynamic in signal characteristics while the latter is generally constant. Thus, evaluation of dynamic changes of fluorescence signal after application of an activatable probe is a potential method to differentiate between fluorescence from HMRG on cancer foci and autofluorescence in surrounding normal tissue over a wide range of dye concentrations.

In this study, we used the sprayable activatable probe, gGlu-HMRG in an animal model of peritoneal ovarian cancer metastases (POCM) and acquired dynamic fluorescence images at varying concentrations of the dye. Using dynamic images we created kinetic maps based on calculated three parameters (Figure 1), maximum fluorescence signal (MF), wash-in rate (WIR), and area under the curve (AUC), which are frequently used as semiquantitative parameters in conventional dynamic imaging of contrast-enhanced MRI and nuclear medicine especially for differentiating between benign and malignant tumors [19-22]. MF is the maximum fluorescence signal observed during the entire dynamic images. WIR is the maximum slope approaching the MF. AUC is the area measured under the time-fluorescence curve. We investigated the utility of kinetic maps to differentiate between cancer foci and background tissue.

\section{RESULTS}

To simulate the effects of dilution, a variety of concentrations of gGlu-HMRG were utilized resulting in the following observations:

\section{5 uM gGlu-HMRG}

Fluorescence intensity of cancer foci increased gradually over $30 \mathrm{~min}$ after spraying gGlu-HMRG on the specimen $(p=0.09$ at $5 \mathrm{~min}$ and $<0.01$ at 10,20 , and $30 \mathrm{~min}$ after gGlu-HMRG, respectively). On the other hand, fluorescence intensity of the small intestine did not change after spraying gGlu-HMRG and there was no significant difference $(p=0.41,0.47,0.41$ and 0.30 at 5, 10, 20 and $30 \mathrm{~min}$ after spraying gGlu-HMRG, respectively) (Figure 2, Supplementary Figures S1, S2 and Supplementary Video S1).

On unprocessed images, the fluorescence intensity of cancer foci was significantly higher than that of small intestine only at $30 \mathrm{~min}$ after spraying gGlu-HMRG ( $p=0.42,0.63,0.08$ and 0.04 at $5,10,20$ and $30 \mathrm{~min}$ after spraying gGlu-HMRG, respectively). On the other hand, maximum fluorescence signal $(\mathrm{MF})$, wash-in rate (WIR), and area under the curve (AUC) were significantly higher in cancer foci than small intestine at all time points even as early as 5 minutes $(p<0.01$ at all time point for all three parameters) (Figures 2, 7, and Supplementary Figures S3-S6).

\section{5 uM gGlu-HMRG}

Fluorescence intensity of cancer foci increased gradually up to $30 \mathrm{~min}$ after spraying gGlu-HMRG $(p=0.30$ and 0.04 at 5 and $10 \mathrm{~min},<0.01$ at 20 and 30 min after spraying gGlu-HMRG, respectively). On the other hand, the fluorescence intensity of small intestine did not change after spraying gGlu-HMRG and there were no significant differences $(p=0.76,0.75,0.69$ and 0.60 at 5, 10, 20 and $30 \mathrm{~min}$ after spraying gGlu-HMRG, respectively) (Figure 3, Supplementary Figures S1, S2 and Supplementary Video S2).

On unprocessed images the fluorescence intensity of cancer foci was significantly higher than that of small intestine only at $30 \mathrm{~min}$ after spraying gGlu-HMRG $(p=0.20,0.75,0.15$ and 0.02 at $5,10,20$ and $30 \mathrm{~min}$ 


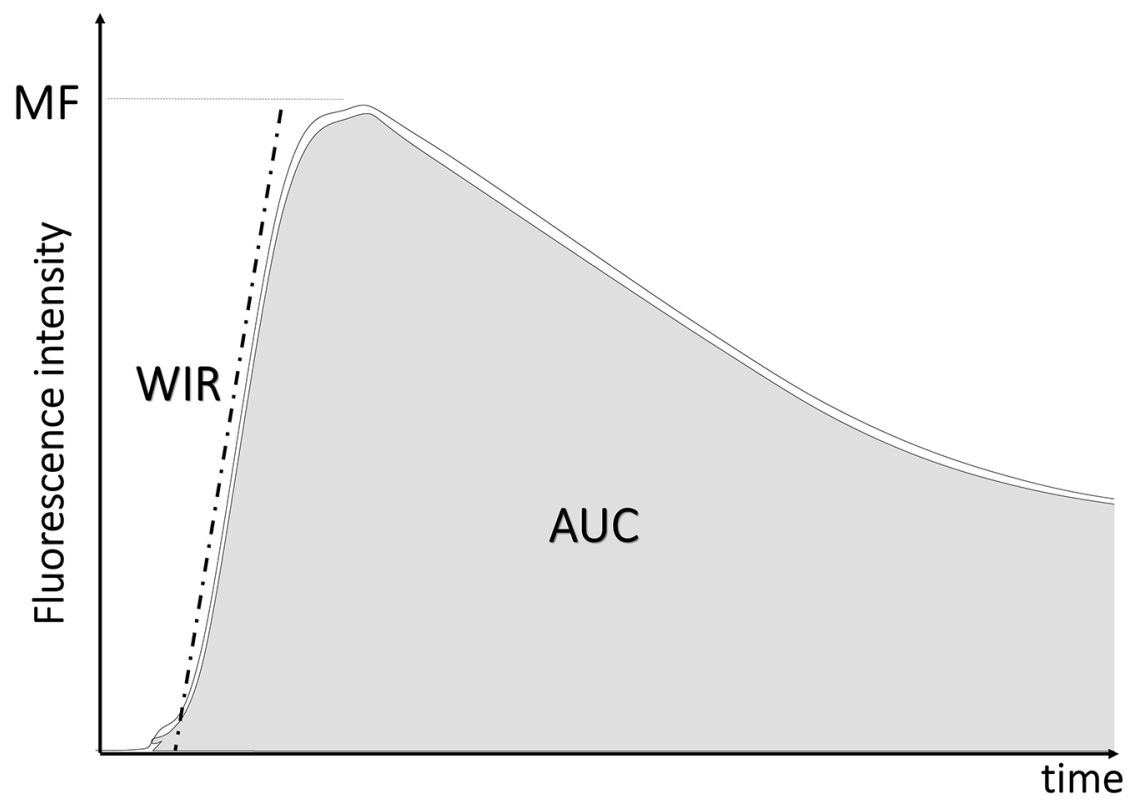

Figure 1: Schema of creating kinetic maps.

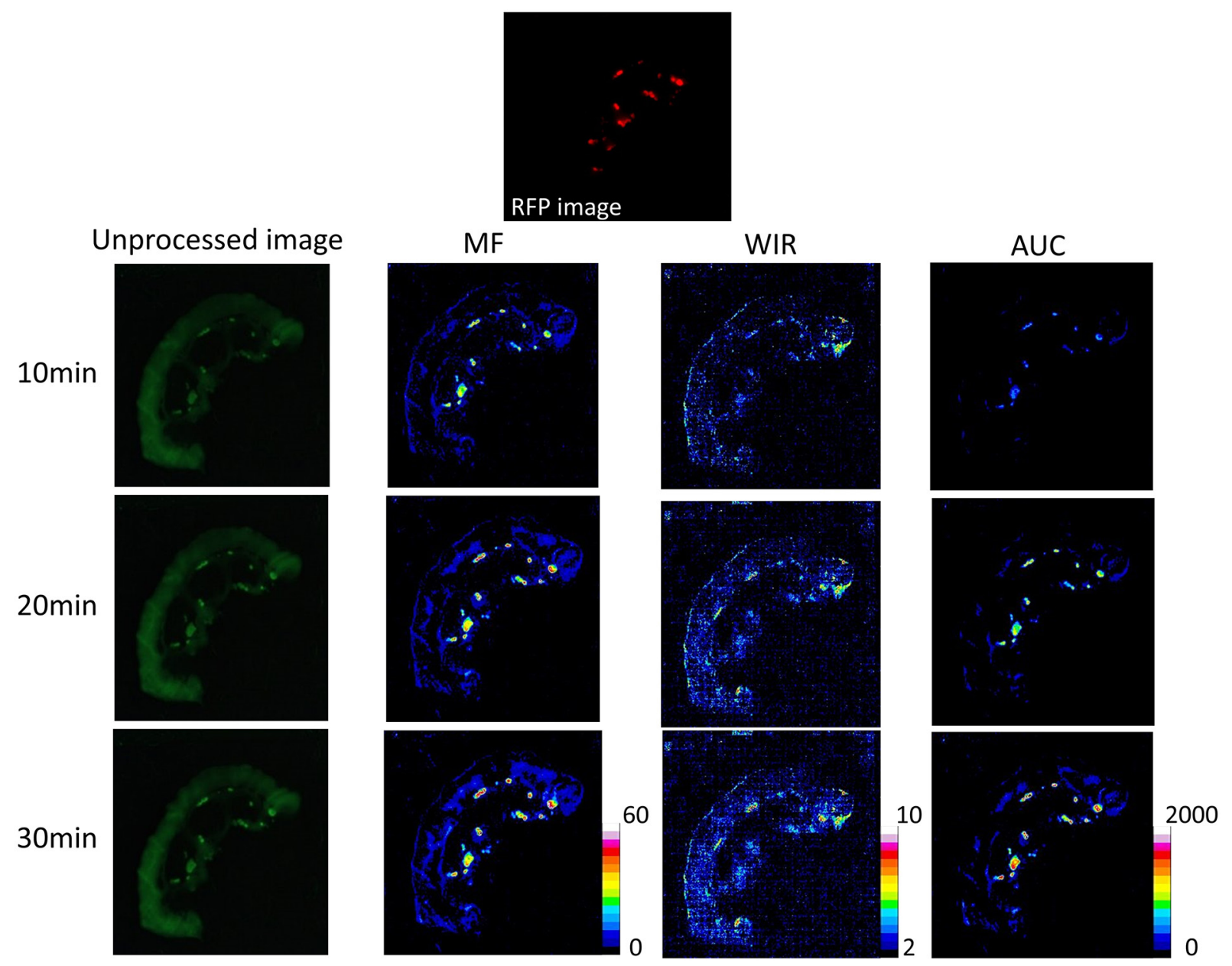

Figure 2: Unprocessed images and kinetic maps (MF, WIR, and AUC maps) using $2.5 \mu \mathrm{M}$ gGlu-HMRG, and RFP image (the standard of reference for cancer location). The detection of cancer foci was more straightforward on MF and AUC maps compared to unprocessed images. 
after spraying gGlu-HMRG, respectively). On the other hand, MF of cancer foci was significantly higher than that of small intestine at all time points $(p=0.02$ at 5 min and $p<0.01$ at 10,20, and $30 \mathrm{~min}$, respectively). AUC of cancer foci was significantly higher than that of small intestine at time points $10 \mathrm{~min}$ and greater $(p=0.15$ at $5 \mathrm{~min}$ and $p<0.01$ at 10,20 , and $30 \mathrm{~min}$ after spraying gGlu-HMRG, respectively). WIR of cancer foci tended to be higher compared to that of small intestine. However, there was no significant difference $(p=0.52,0.75,0.26$ and 0.07 at 5, 10, 20 and $30 \mathrm{~min}$ after spraying gGluHMRG, respectively) (Figures 3, 8 and Supplementary Figures S3-S6).

\section{0 uM gGlu-HMRG}

Fluorescence intensity of cancer foci increased gradually up to $30 \mathrm{~min}$ after spraying gGlu-HMRG $(p=0.47$ and 0.05 at 5 and $10 \mathrm{~min},<0.01$ at 20 and $30 \mathrm{~min}$ after spraying probe, respectively). On the other hand, fluorescence intensity of the small intestine did not change after spraying gGlu-HMRG and there were no significant differences $(p=0.79,0.81,0.77$ and 0.77 at 5, 10, 20 and $30 \mathrm{~min}$ after spraying gGlu-HMRG, respectively) (Figure 4, Supplementary Figures S1, S2 and Supplementary Video S3).

On unprocessed images fluorescence intensity of cancer foci was significantly higher than that of small intestine only at $30 \mathrm{~min}$ after spraying gGlu-HMRG ( $p=0.63,0.42,0.11$ and 0.01 at $5,10,20$ and $30 \mathrm{~min}$ after spraying gGlu-HMRG, respectively). On the other hand, MF and AUC of cancer foci was significantly higher than that of small intestine at all time points $(p=0.02$ at 5 min and $p<0.01$ at 10,20 , and $30 \mathrm{~min}$ after spraying gGlu-HMRG for MF, $p=0.02$ at $5 \mathrm{~min}$ and $p<0.01$ at 10, 20, and $30 \mathrm{~min}$ after spraying gGlu-HMRG for AUC, respectively). WIR of cancer foci tended to be higher compared to that of small intestine with a significant
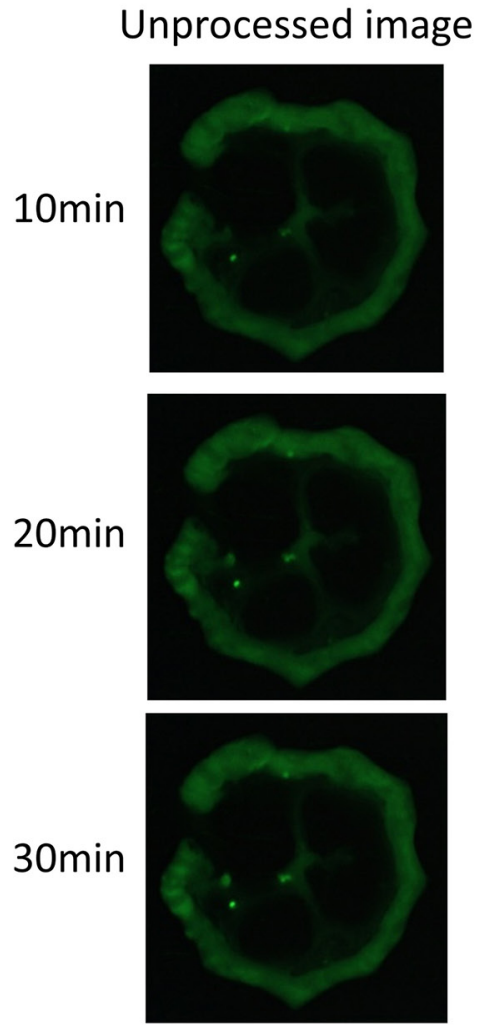
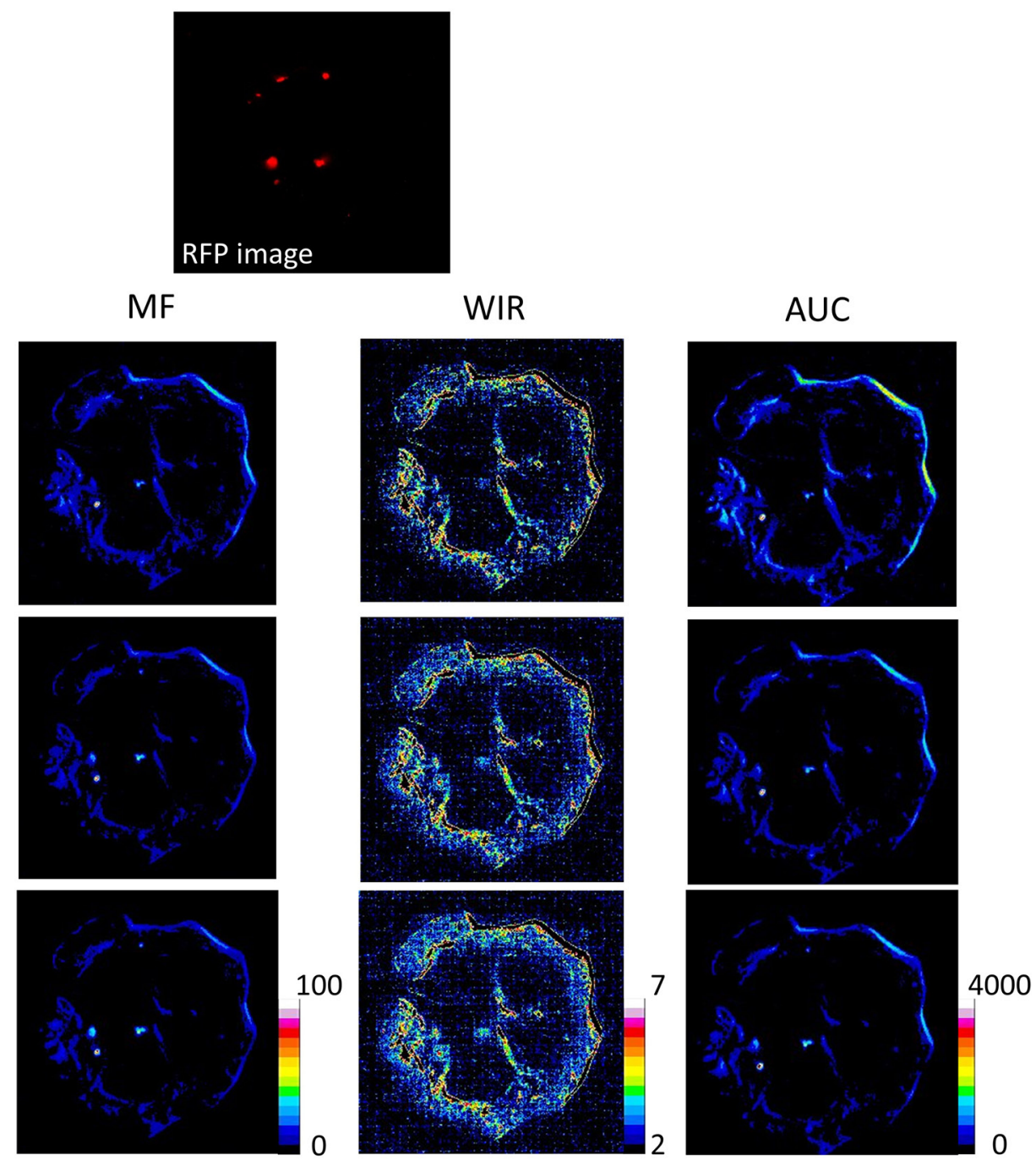

Figure 3: Unprocessed images and kinetic maps (MF, WIR, and AUC maps) using $5 \mu \mathrm{M}$ gGlu-HMRG, and RFP image (the standard of reference for cancer location). The detection of cancer foci was a little easier on MF and AUC maps compared to unprocessed images. 
difference at 20 and $30 \mathrm{~min}$ after spraying gGlu-HMRG ( $p=0.20,0.08,0.04$ and 0.02 at 5, 10, 20 and 30 min after spraying gGlu-HMRG, respectively) (Figures 4, 9 and Supplementary Figures S3-S6).

\section{0 uM gGlu-HMRG}

Fluorescence intensity of cancer foci increased gradually up to $30 \mathrm{~min}$ after spraying gGlu-HMRG $(p=0.22$ and 0.01 at 5 and $10 \mathrm{~min},<0.01$ at 20 and 30 min after spraying gGlu-HMRG, respectively). On the other hand, fluorescence intensity of the small intestine did not change after spraying gGlu-HMRG and there was no significant difference $(p=0.69,0.71,0.70$ and 0.69 at 5, 10, 20 and $30 \mathrm{~min}$ after spraying gGlu-HMRG, respectively) (Figure 5, Supplementary Figures S1, S2 and Supplementary Video S4).

On unprocessed images fluorescence intensity of cancer foci tended to be higher than that of small intestine with significant differences at 20 and 30 min after spraying gGlu-HMRG $(p=0.52$ and 0.33 at 5 and $10 \mathrm{~min},<0.01$ at 20 and $30 \mathrm{~min}$ after spraying gGlu-HMRG, respectively).
All three parameters, MF, WIR and AUC of cancer foci were significantly higher than that of small intestine at all time points ( $p<0.01$ at all time points for all three parameters) (Figures 5, 10 and Supplementary Figures S3-S6).

\section{0 uM gGlu-HMRG}

Spraying gGlu-HMRG resulted in a marked increase of fluorescence intensity of cancer foci followed by gradual increase up to $30 \min$ ( $p<0.01$ at all time points). On the other hand, fluorescence intensity of small intestine did not change after spraying gGlu-HMRG and there were no significant differences $(p=0.41,0.47,0.41$ and 0.30 at 5 , 10, 20 and $30 \mathrm{~min}$ after spraying gGlu-HMRG, respectively) (Figure 6, Supplementary Figures S1, S2 and Supplementary Video S5).

On unprocessed images fluorescence intensity of cancer foci was higher than that of small intestine with significant differences at all time points $(p<0.01$ at all time points). All three parameters, MF, WIR and AUC of cancer foci were also significantly higher than that of small intestine at all time points $(p<0.01$ at all time points

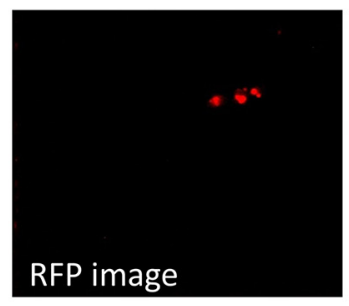

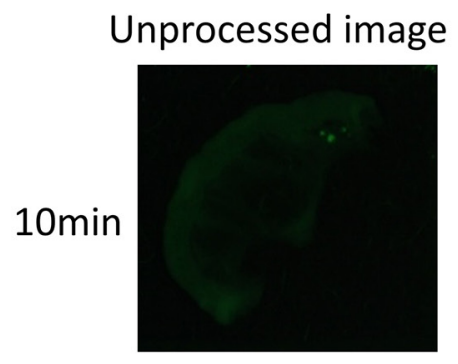
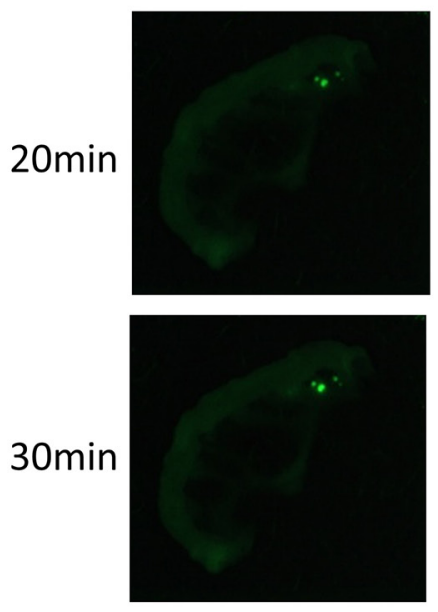

MF
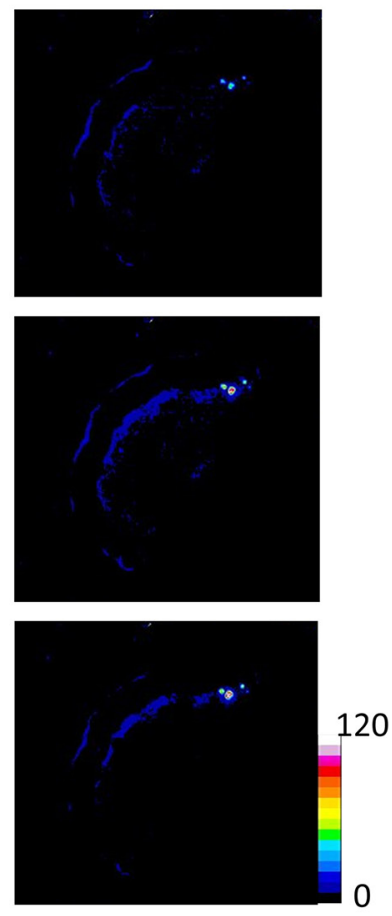

WIR
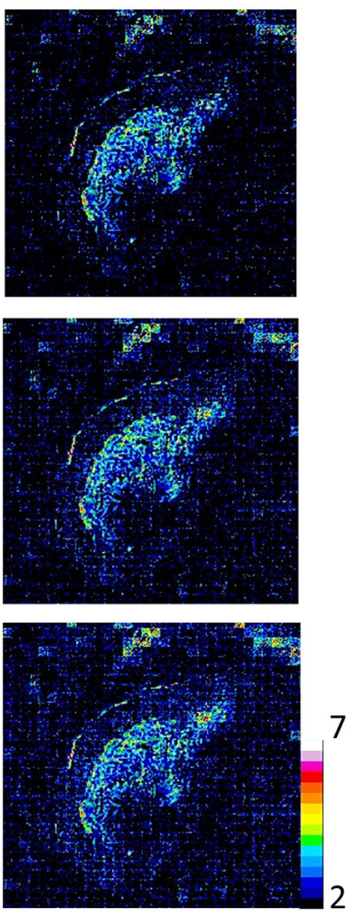

AUC
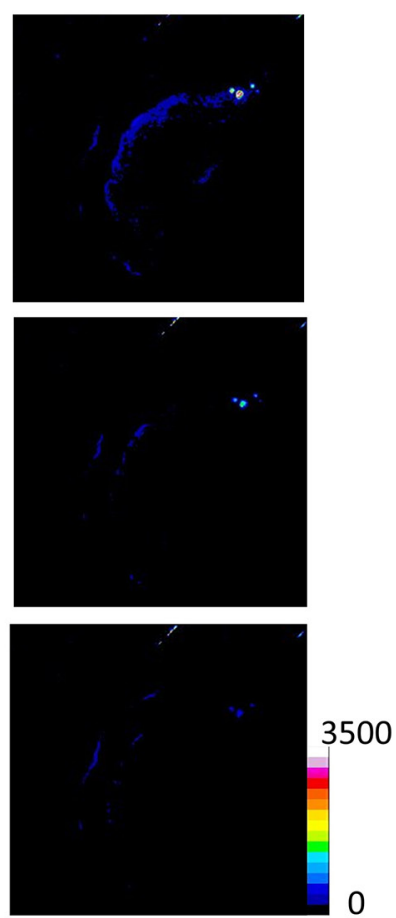

Figure 4: Unprocessed images and kinetic maps (MF, WIR, and AUC maps) using $10 \mu$ M gGlu-HMRG, and RFP image (the standard of reference for cancer location). The detection of cancer foci on unprocessed images and kinetic maps was almost identical visually. 
for all three parameters) (Figures 6, 11 and Supplementary Figures S3-S6). However, high signal on small bowel mesentery hampered the evaluation of cancer foci on the WIR color map.

\section{Comparison of tumor: small intestine ratio at an early time point}

Tumor:small intestine ratio (T:SI) of unprocessed images was approximately zero up to $20 \mu \mathrm{M}$. On the other hand, T:SI of the three parameters tended to demonstrate higher ratios compared to ratios of unprocessed images regardless of concentration of gGlu-HMRG (Figure 12). Using $2.5 \mu \mathrm{M}$ and $20 \mu \mathrm{M}$ gGlu-HMRG, the T:SI using $\mathrm{MF}$ and AUC was significantly higher than the T:SI using unprocessed images $(p=0.03,0.68$ and $<0.01$ for $\mathrm{MF}$, WIR, and AUC at $2.5 \mu \mathrm{M}$, and $p=0.05,0.52$ and $<0.01$ for MF, WIR, and AUC at $20 \mu \mathrm{M}$, respectively). Using $5 \mu \mathrm{M}$ gGlu-HMRG the T:SI of the AUC was significantly higher than that of unprocessed images $(p=0.35,0.71$ and 0.04 for MF, WIR, and AUC, respectively). Using
$10 \mu \mathrm{M}$ and $100 \mu \mathrm{M}$ gGlu-HMRG there was no significant difference in T:SI for all three parameters compared to that of unprocessed images $(p=0.63,0.74$ and 0.15 for $\mathrm{MF}$, WIR, and AUC at $10 \mu \mathrm{M}$, and $p=0.29,0.70$ and 0.05 for $\mathrm{MF}, \mathrm{WIR}$, and AUC at $100 \mu \mathrm{M}$, respectively).

\section{DISCUSSION}

At all concentrations, fluorescence intensity of HMRG in cancer foci increased continually over $30 \mathrm{~min}$ after spraying gGlu-HMRG on the surface. Meanwhile, fluorescence intensity of the small intestine did not change. These results indicate that gGlu-HMRG was specifically activated by cancer even at low concentrations and was specifically activated by the tumor microenvironment even at high concentrations.

On unprocessed images, the fluorescence intensity of cancer foci was significantly higher than that of small intestine only at $30 \mathrm{~min}$ after spraying gGlu-HMRG when the concentration of the dye was up to $10 \mu \mathrm{M}$. This is likely due to the fact that at lower concentrations

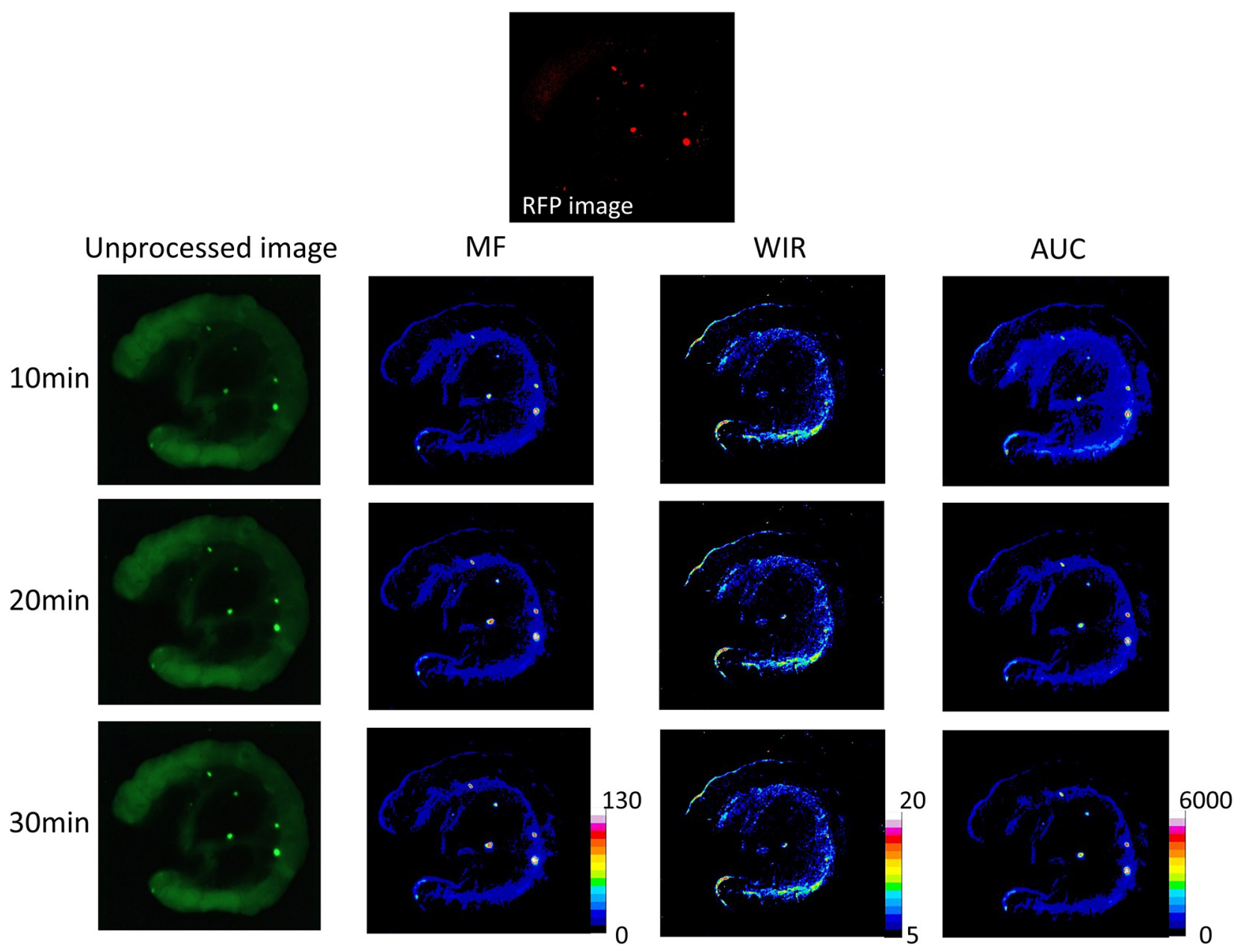

Figure 5: Unprocessed images and kinetic maps (MF, WIR, and AUC maps) using $20 \mu M$ gGlu-HMRG, and RFP image (the standard of reference for cancer location). The detection of cancer foci on unprocessed images and kinetic maps was almost identical visually. However, autofluorescence of small intestine was prominent on unprocessed images. 
of the dye fluorescence was comparable in intensity to autofluorescence but above $10 \mu \mathrm{M}$ the dye produced stronger fluorescence compared to autofluorescence.

However, kinetic maps were able to discern the presence of HMRG within cancer foci even at lower concentrations of the dye. For instance, the MF map showed that cancers were significantly higher in signal than small intestine at all time points even using $2.5 \mu \mathrm{M}$, the lowest concentration of gGlu-HMRG in the study. The AUC map also showed that cancers were significantly higher than small intestine at all time points. Thus, the dynamic parameters $\mathrm{MF}$ and $\mathrm{AUC}$, were useful in overcoming the effects of autofluorescence and may be especially useful with low concentrations of gGlu-HMRG as might occur due to uneven dilution in fluids pooling in a body cavity.

The WIR map tended to demonstrate higher signal in cancers compared to small intestine regardless of concentration of gGlu-HMRG. However, the WIR produced inconsistent results at several time points indicating it is a less robust parameter than MF and AUC. For instance, there was no significant difference in WIR between cancer foci and small intestine at all time points with $5 \mu \mathrm{M}$ gGlu-HMRG. WIR is reflective of the maximum slope of fluorescence intensity curve on subtracted images and may reflect presence of GGT within the tumor [9]. The exact function of GGT and how its presence benefits the tumor is still unclear [23]. However, GGT has also been reported to be overexpressed in several human tumors, including those from cervical and ovarian cancers [9-12] and is thought to promote tumor progression, invasion, and drug resistance, possibly through modulation of the intracellular redox metabolism [24]. Thus, WIR has the potential to be an imaging biomarker for GGT expression but may be less useful as a means of detecting low levels of HMRG.

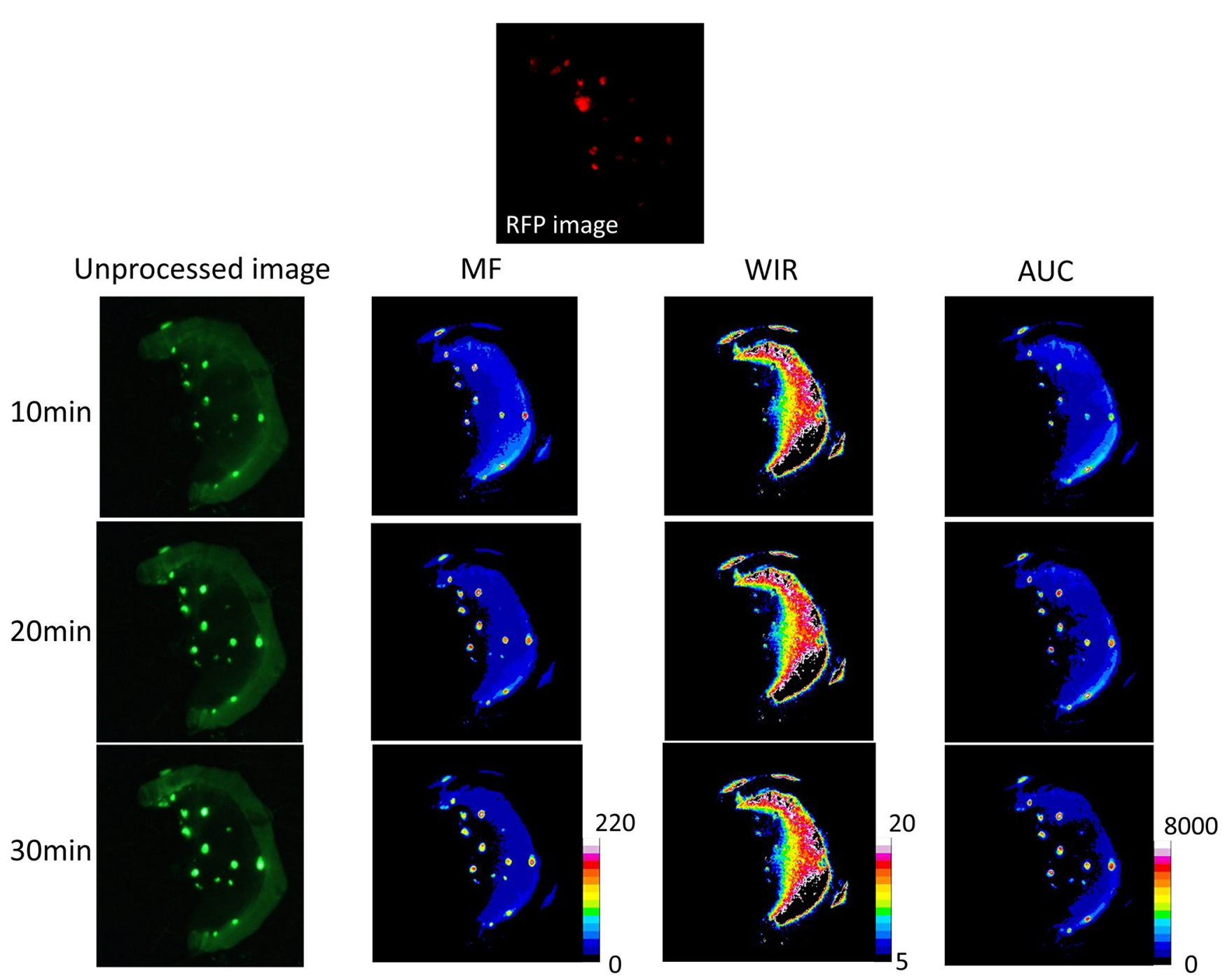

Figure 6: Unprocessed images and kinetic maps (MF, WIR, and AUC maps) using $100 \mu$ MGlu-HMRG, and RFP image (the standard of reference for cancer location). Cancer foci were clearly detected on the unprocessed images. On the other hand, small bowel mesentery showed high fluorescence signal on WIR maps potentially obscuring some lesions. 


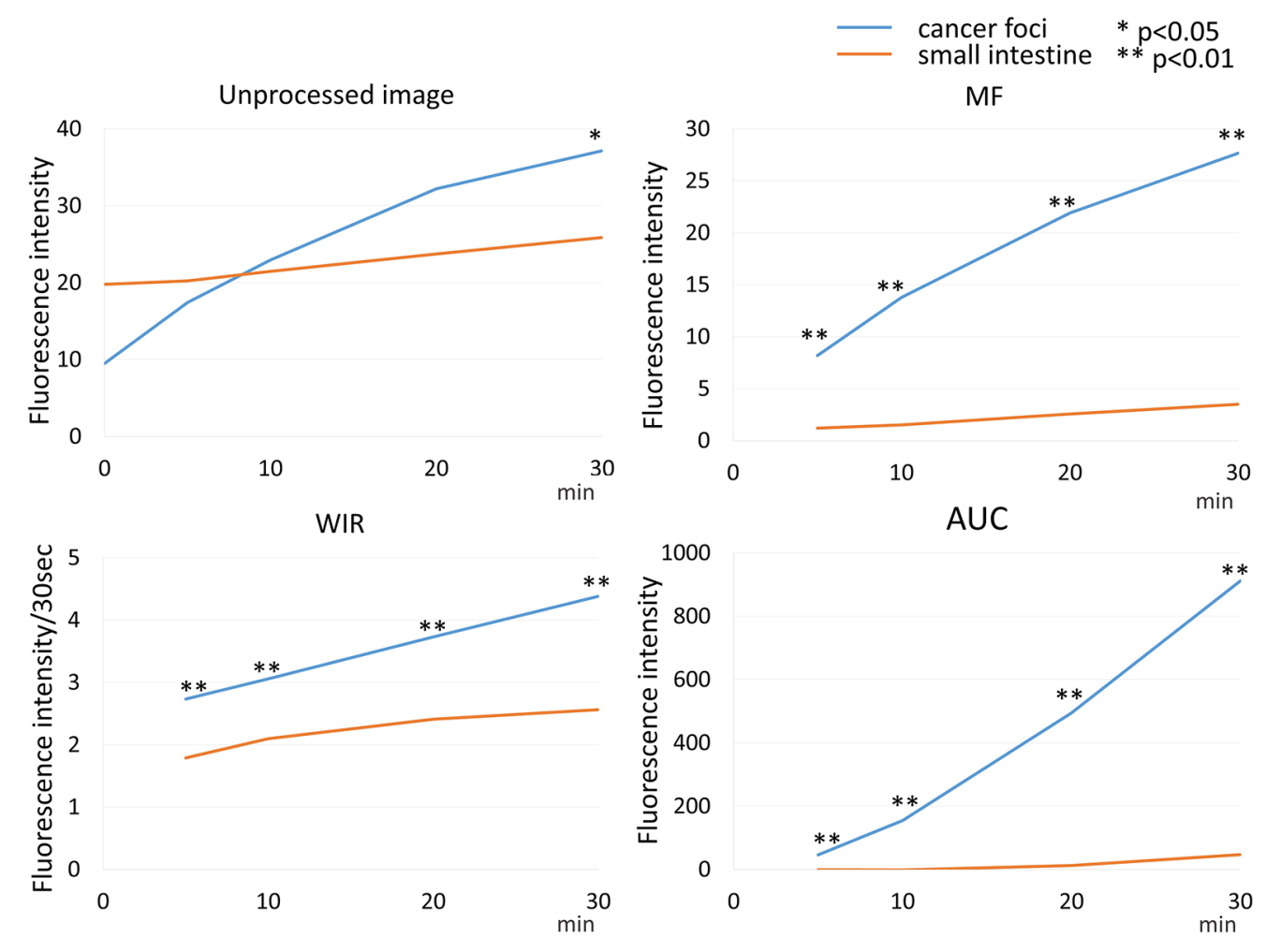

Figure 7: Time fluorescence intensity curve of the cancer foci and small intestine on unprocessed images, MF, WIR, and AUC maps using $2.5 \mu \mathrm{M}$ gGlu-HMRG. Difference between cancer foci and small intestine was examined at each time point.
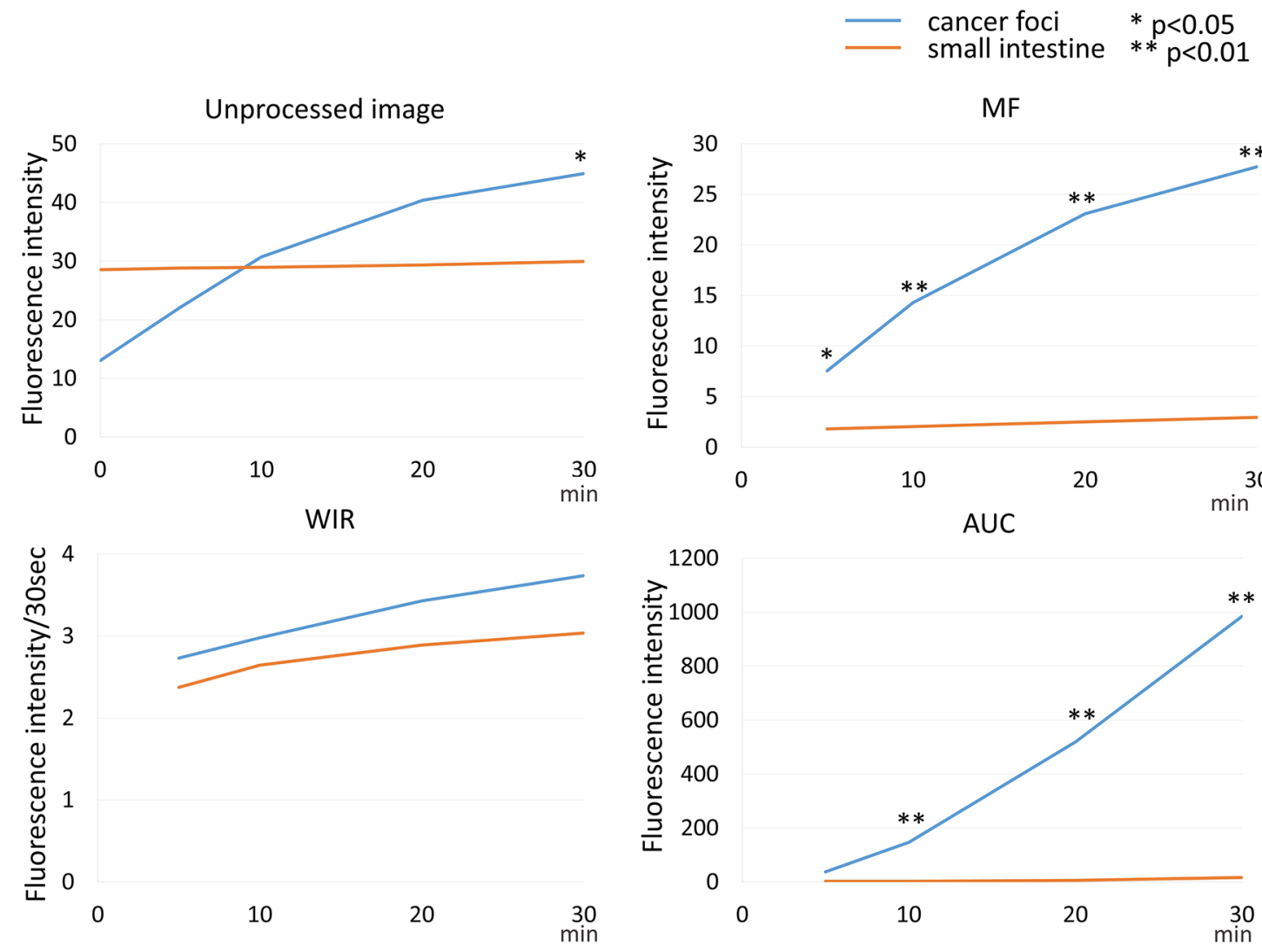
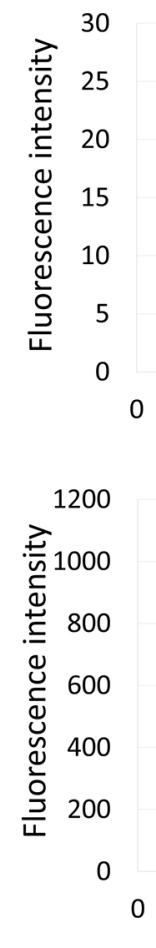
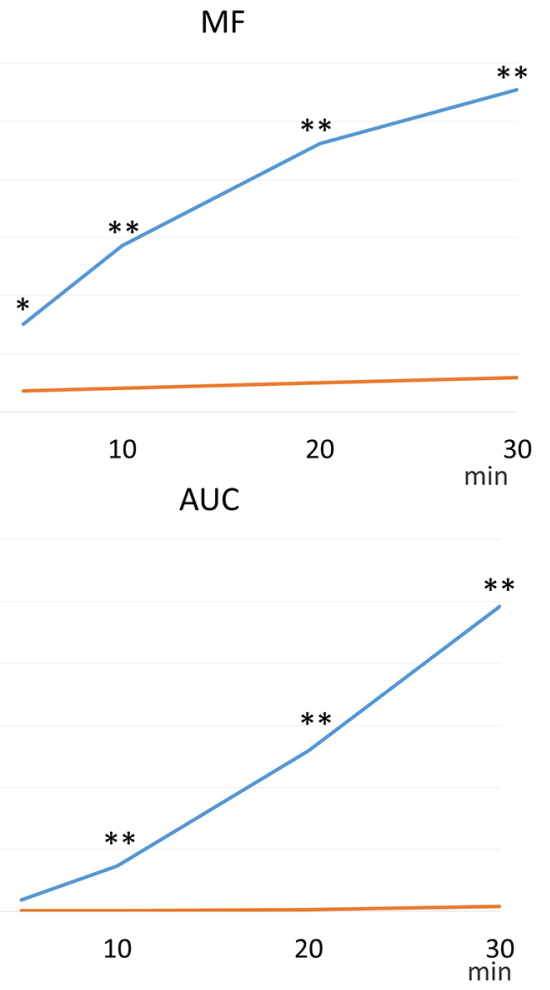

Figure 8: Time fluorescence intensity curve of the cancer foci and small intestine on unprocessed images, MF, WIR, and AUC maps using $5 \mu$ M gGlu-HMRG. Difference between cancer foci and small intestine was examined at each time point. 

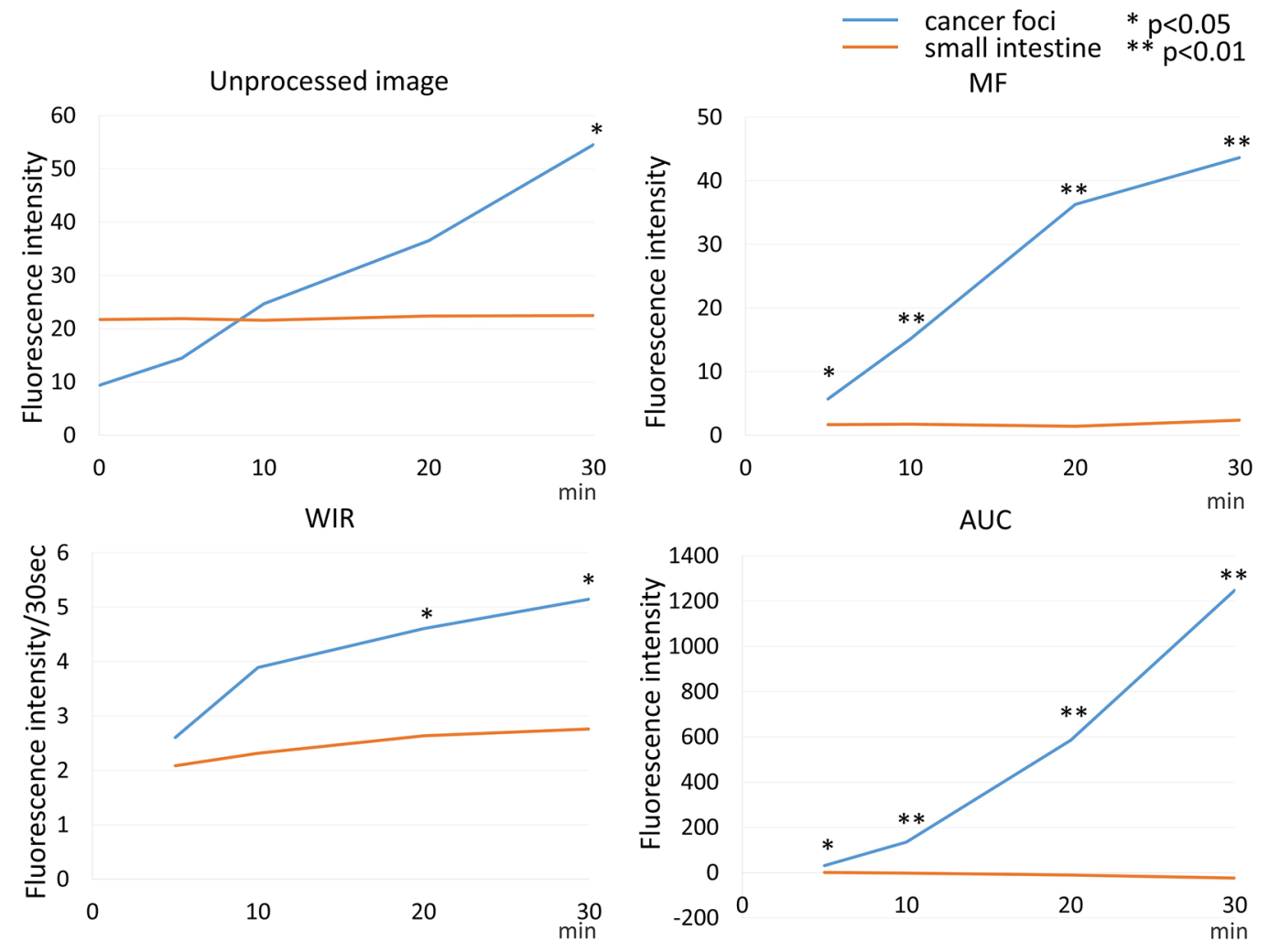

Figure 9: Time fluorescence intensity curve of the cancer foci and small intestine on unprocessed images, MF, WIR, and AUC maps using $10 \mu \mathrm{M}$ gGlu-HMRG. Difference between cancer foci and small intestine was examined at each time point.
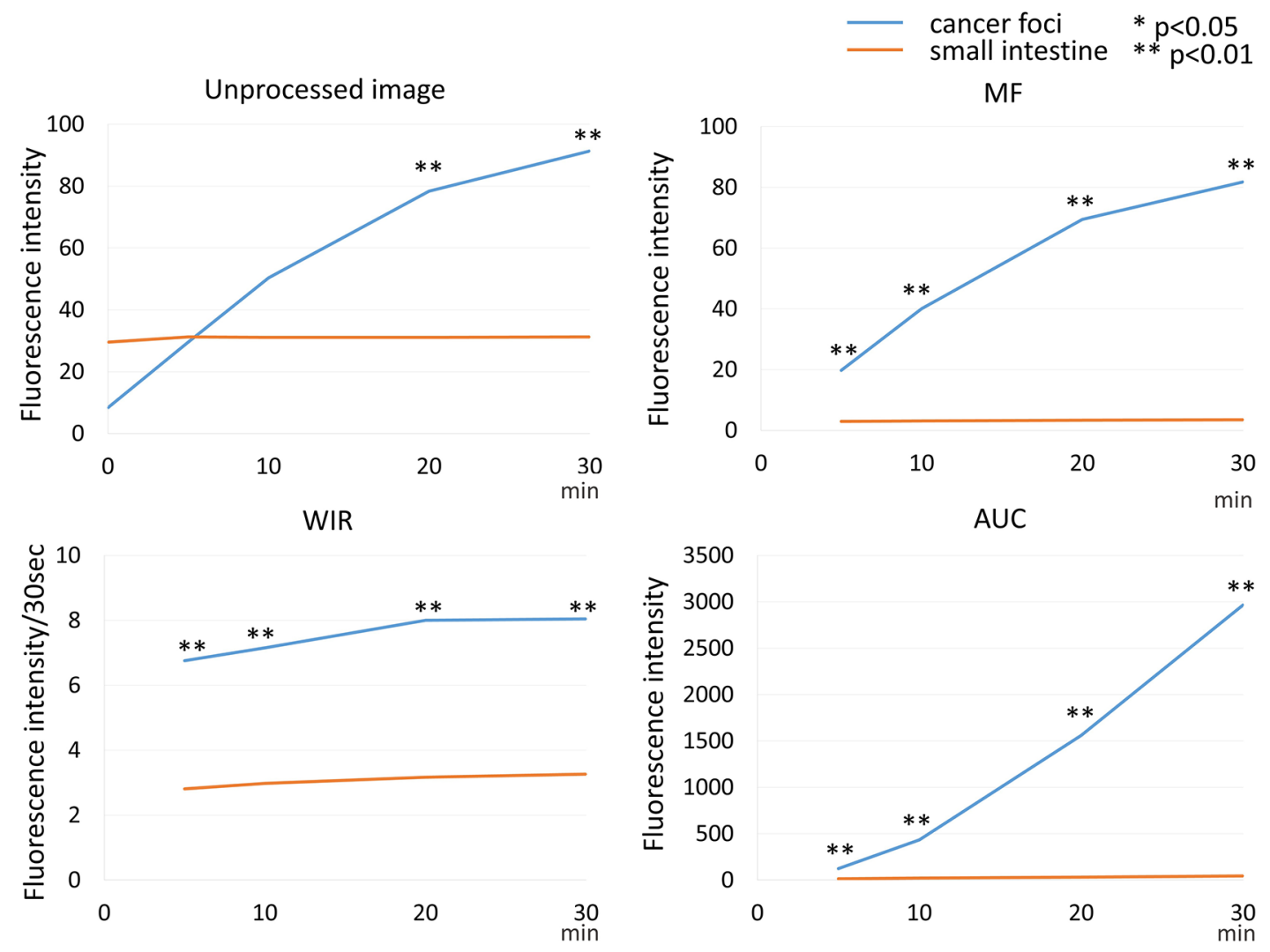

Figure 10: Time fluorescence intensity curve of the cancer foci and small intestine on unprocessed images, MF, WIR, and AUC maps using $20 \mu \mathrm{M}$ gGlu-HMRG. Difference between cancer foci and small intestine was examined at each time point. 
When the highest concentration, $100 \mu \mathrm{M}$ gGluHMRG, was used, cancer foci could be discerned at all time points on unprocessed images. The MF and AUC maps were also positive but were superfluous to the unprocessed images. The WIR map had high overall signal hampering the detection of cancer foci thus, confirming it is the least useful kinetic parameter. The gGlu-HMRG solution with high concentration could be activated by a minimal amount of GGT extruded from cancer foci to adjacent mesentery and emit fluorescence signal on mesentery shortly after spraying gGlu-HMRG. Attention must be paid to this issue when using the WIR map with high concentration gGlu-HMRG.

Thus, these data suggested that kinetic maps, especially those based on MF and AUC, permitted detection of cancer foci across a wide range of dye concentrations including low concentrations where unprocessed images were unhelpful at early time points. At high concentrations of gGlu-HMRG cancer foci could be clearly discerned on the unprocessed images. Thus, unprocessed images are most useful at high concentrations and kinetic maps are most useful at low concentrations and their combined use is therefore, complementary given that the dye is likely to get diluted in an unpredictable manner due to pools of fluid in body cavities (e.g. ascites, irrigation fluid etc.). These findings are of importance in improving the performance of dyes used in image guided surgical or endoscopic procedures. Most probes, including gGlu-HMRG, have been developed to aid surgeons in detecting tiny cancer foci, delineating the borders of tumors for complete removal and confirming the absence of residual tumor $[9,14-16]$. The probe may be distributed on the surface of the target tissue in an inhomogeneous manner and the concentration of probe may vary with location. Thus, the combination of unprocessed images and kinetic maps, which detect cancer foci regardless of dye concentration, is considered to be a superior method for detection of cancer foci clinically.

Classically, fluorescence from a specific probe has been detected with multispectral imaging, which is the most sensitive optical technique for the identification of target tumors. However, current multispectral imaging
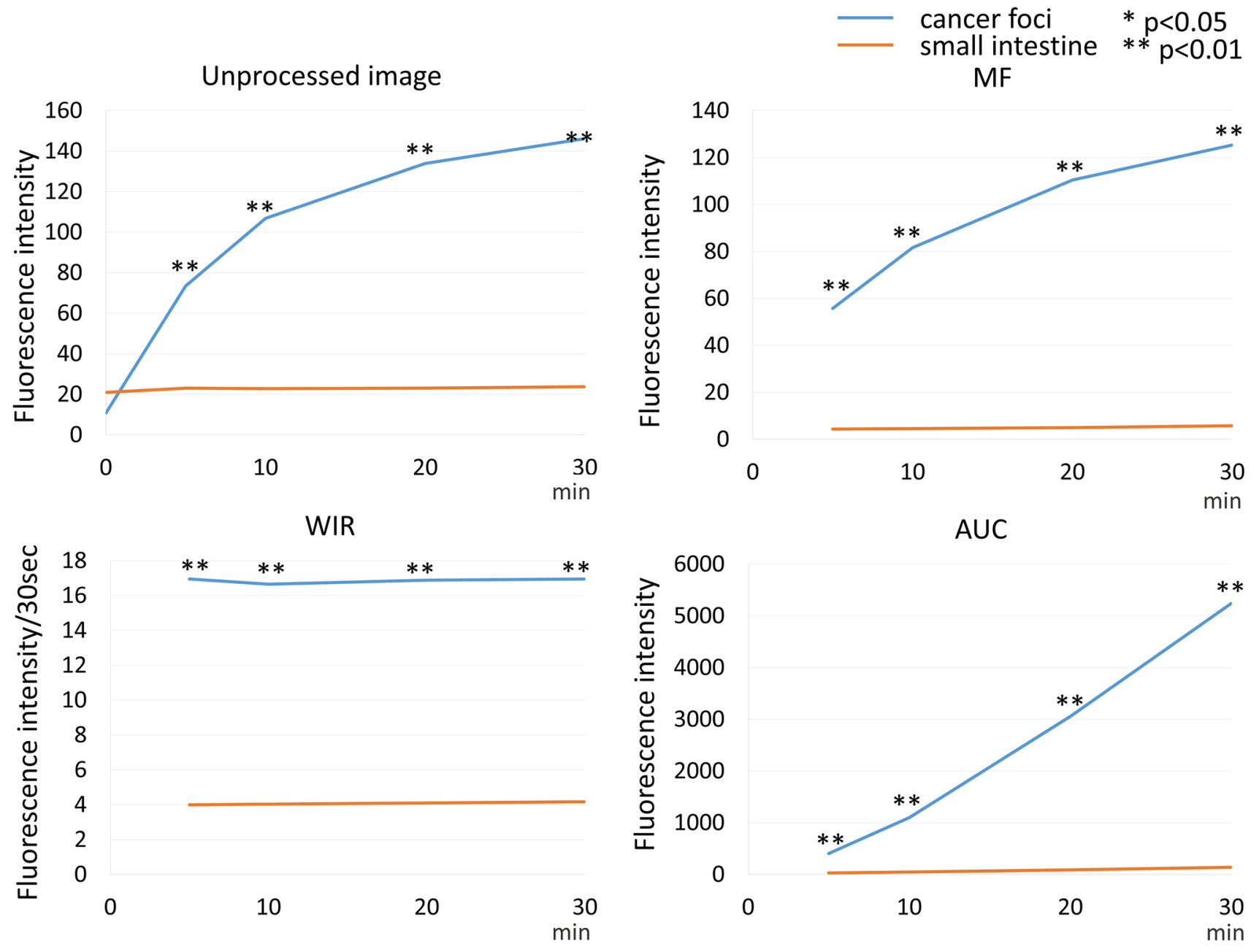

Figure 11: Time fluorescence intensity curve of the cancer foci and small intestine on unprocessed images, MF, WIR, and AUC maps using $100 \mu \mathrm{M}$ gGlu-HMRG. Difference between cancer foci and small intestine was examined at each time point. 
takes at least 10 seconds per frame using expensive specially-resolved filter devices for scanning sufficient range of spectra and therefore, is not amenable to surgical or endoscopic procedures [6, 18]. Kinetic parametric maps, such as MF or AUC, offer superior contrast between tumor and small intestine before target tumors show up with sufficient fluorescence intensity on unprocessed images. Thus, this simple image processing of dynamic fluorescence imaging can assist tumor detection on unprocessed images obtained by inexpensive regular video camera. Moreover, kinetic maps can be created almost real-time with an appropriate image processing program because of the simple processing, suggesting that they could be useful for detection of hard-to-see tumors in intraoperative setting.

A reported approach for reducing autofluorescence is to use narrow bandwidth filters [25-29]. In this camera system, we employed a similar narrow bandwidth filter setting that is appropriate for excitation and emission of HMRG. However, when applied low concentration of gGlu-HMRG, signal from tumors was difficult to be detected especially at short time after administration because the signal from gGlu-HMRG was quickly increasing but still lower than that from fluorescent proteins including green fluorescent protein (GFP) which emits strong fluorescence signal [30-39]. Kinetic maps helped detecting such hard-to-see HMRG signal yielded from tumors with regular video cameras. Thus, these kinetic maps are useful especially for detecting low fluorescence signal derived from probe submerged in autofluorescence of surrounding normal tissue.

One important limitation in kinetic maps is the mis-registration. Kinetic maps are created by processing serial images which are taken different time after spraying probes. Perfect subtraction is sometimes difficult because of the slight movement of fresh samples at each time point due to natural contraction or evaporation of fluid. Incomplete subtraction especially at the edge of fluorescent objects might cause a potential error on post-processed parameters because of a large change in fluorescence signal. Indeed, cancer foci adjacent to small intestine confirmed on RFP images was sometimes difficult to
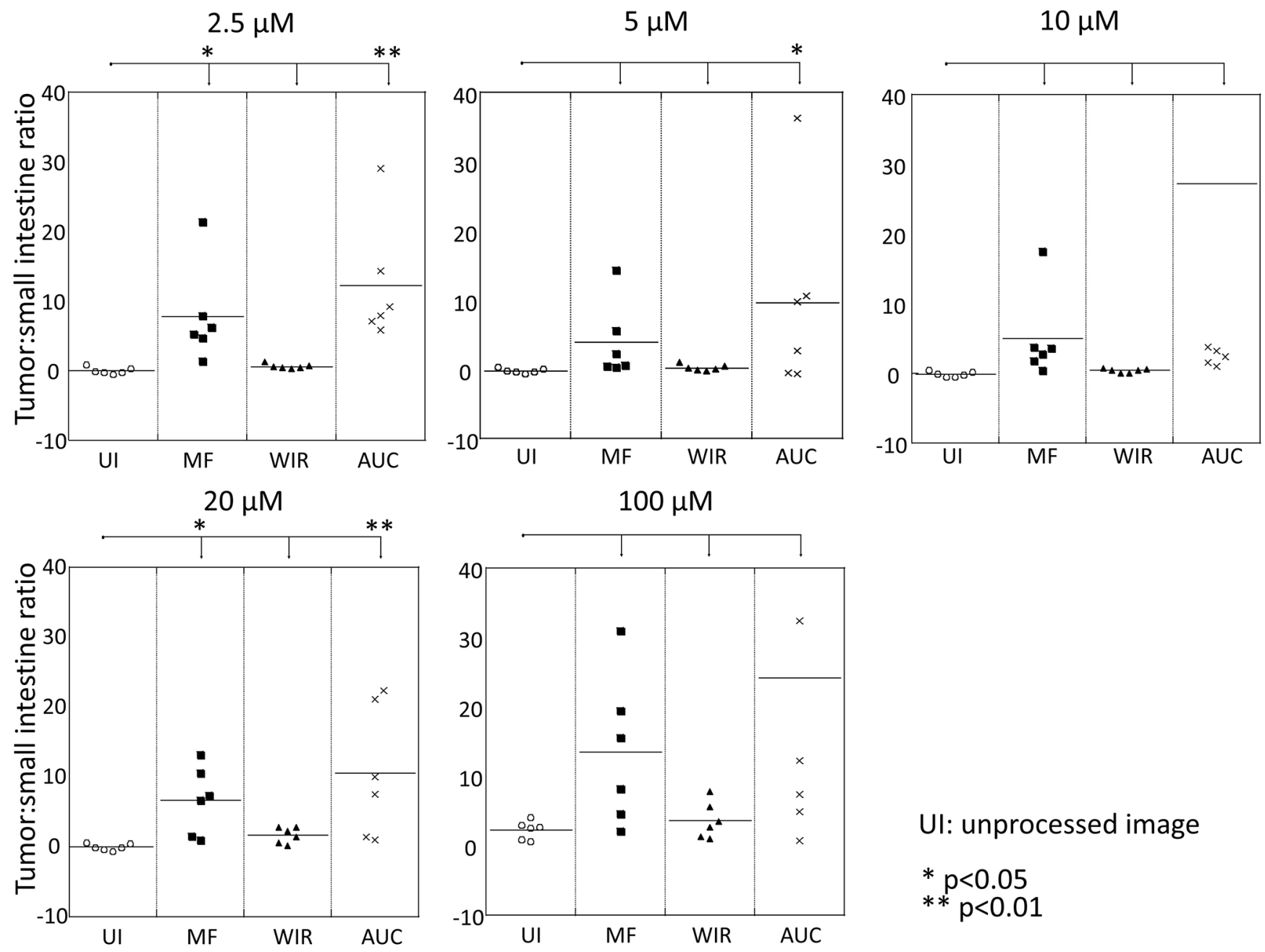

Figure 12: Comparison of tumor:small intestine ratio of three parameters, MF, WIR, and AUC, compared to that of unprocessed image. 
detect on kinetic maps when using low concentration of gGlu-HMRG because false high values on kinetic maps around small intestine due to mis-registration hampered the detection of cancer foci (see Figure 3). Attention must be paid to this issue when a kinetic map is read.

In conclusion, kinetic maps were useful in overcoming autofluorescence especially with low concentrations of gGlu-HMRG. While cancer foci were detected clearly on unprocessed images at high concentrations of gGlu-HMRG, kinetic maps were superior when low concentrations of the dye were present. Thus, the combination of unprocessed images and kinetic maps is potentially important for detecting cancer foci regardless of concentration of gGluHMRG during surgical or endoscopic procedures.

\section{MATERIALS AND METHODS}

\section{Reagents}

gGlu-HMRG, a rapidly activatable cancer-selective fluorescence imaging probe, was synthesized as described previously [9].

\section{Cell lines and culture}

SHIN3, is an ovarian cancer cell line that highly expresses GGT and shows strongly positive fluorescent signal with gGlu-HMRG [9]. SHIN3-DsRed contains a red fluorescent protein (RFP DsRed2)-expressing plasmid (Clontech Laboratories) that was stably transfected into SHIN3 cells to enable detection on ex vivo optical imaging of POCM [40]. Cell lines were grown in RPMI 1640 supplemented with $10 \% \mathrm{FBS}$ and $1 \%$ penicillin-streptomycin in tissue culture flasks in a humidified incubator at $37^{\circ} \mathrm{C}$ in an atmosphere of 95\% air and 5\% carbon dioxide.

\section{Animal model}

All procedures were performed in compliance with the Guide for the Care and Use of Laboratory Animals [41] and approved by the local Animal Care and Use Committee. Six- to 8-week old female homozygote athymic nude mice were purchased from Charles River (National Cancer Institute, Frederick, MD).

To generate the animal model, intraperitoneal xenografts were established by intraperitoneal (i.p.) injection of $2 \times 10^{6}$ SHIN3-DsRed cells suspended in 200 to $300 \mu \mathrm{l}$ of phosphate-buffered saline (PBS) into the peritoneal cavity of nude mice. Imaging was performed at 14-21 days after injection of the cells.

\section{Ex vivo activatable imaging}

gGlu-HMRG stock solution (containing $0.5 \% \mathrm{v} / \mathrm{v}$ DMSO as a co-solvent) was suspended in PBS to generate the following concentrations: $2.5,5,10,20$, and $100 \mu \mathrm{M}$ gGlu-HMRG solution which were used to simulate dilution in a clinical situation. Mice with tumors were euthanized by carbon dioxide inhalation. Immediately after euthanasia, the mouse abdominal wall was incised, and the abdominal cavity was exposed. The small bowel, its mesentery and any POCM were extracted en bloc.

To examine the dynamic changes in green fluorescence intensity over time, serial fluorescence imaging was performed after gGlu-HMRG was sprayed on the specimen. A portable fluorescence camera (Discovery INDEC BioSystems, Santa Clara, CA, USA) was utilized [42] with the following filter set: band-pass filter from 450 to $490 \mathrm{~nm}$ for excitation light and from 511 to 551 $\mathrm{nm}$ for emission light, with an exposure time of $50 \mathrm{msec}$. Extracted specimens were placed on a non-fluorescent plate. After baseline images were obtained, a $100 \mu \mathrm{l}$ solution of gGlu-HMRG $(2.5,5,10,20$, and $100 \mu \mathrm{M}$, respectively) was sprayed on a dry specimen surface. Real-time fluorescence images were recorded every $30 \mathrm{sec}$ between 0 and $30 \mathrm{~min}$ after gGlu-HMRG administration.

For evaluation of red fluorescence indicating the presence of tumor, images were acquired using the Maestro In-Vivo Imaging System (Cri Inc.). The following filter set was used: a band-path filter from 503 to $555 \mathrm{~nm}$ for excitation light and a long-pass filter over $645 \mathrm{~nm}$ for emission light. The tunable emission filter was automatically stepped in $10 \mathrm{~nm}$ increments from 600 to $800 \mathrm{~nm}$ at constant exposure times. The spectral fluorescence images consisting of spectra from autofluorescence and RFP were then unmixed, based on their known spectral patterns using commercial software (Maestro software; CRi).

\section{Image analysis}

All images were analyzed using Image $\mathrm{J}$ software (http://rsb.info.nih.gov/ij/). First, using unprocessed images regions of interest (ROIs) were drawn within the tumor nodules depicted by the RFP images (true positive cancer foci) and in the normal adjacent small bowel, and then the average fluorescence intensity of each ROI was calculated. Next, we generated a fluorescence intensity curve using a time series of images. Subtracted images were created by subtracting the pre images (initial images before spraying gGlu-HMRG) from each of the post images (images after spraying gGlu-HMRG). Then, we calculated three parameters from each fluorescence intensity curve using subtracted images: maximum fluorescence signal (MF), wash-in rate (WIR), and area under the curve (AUC). MF is the maximum fluorescence signal observed during the entire dynamic images. WIR (fluorescence intensity/30 sec) is the maximum slope approaching the MF. AUC is the area measured under the time-fluorescence curve (Figure 1). Kinetic maps based on these three parameters, were created. For comparison of their utility for differentiating between cancer foci and background tissue at early time point, we calculated the tumor:small intestine ratio at $5 \mathrm{~min}$ after spraying gGlu-HMRG using following equation:

$$
\mathrm{T}: \mathrm{SI}=\left(\mathrm{TFI}_{5 \min }-\mathrm{SIFI}_{5 \min }\right) /\left|\mathrm{SIFI}_{5 \min }\right|
$$


Where T:SI is the tumor:small intestine ratio, TFI is the fluorescence intensity of tumor at $5 \mathrm{~min}$, SIFI is fluorescence intensity of small intestine at $5 \mathrm{~min}$. We used the absolute value for the small intestine fluorescence intensity because baseline subtracted values may be negative in signal intensity.

\section{Statistical analysis}

Statistical analysis was performed with JMP 10 software (SAS Institute, Cary, NC). We determined the differences in fluorescence intensity at 5, 10, 20, and $30 \mathrm{~min}$ after spraying gGlu-HMRG at various concentrations compared to the starting value and the changes in tumor:small intestine ratio compared to the unprocessed image using Dunnett's multiple comparison. The difference of fluorescence intensity on unprocessed images and three parameter images calculated from subtracted dynamic images between cancer foci and small intestine was determined at 5, 10, 20, and $30 \mathrm{~min}$, respectively. For comparisons of fluorescence intensity between cancer foci and small intestine a two-sided MannWhitney's $U$ test was employed. Differences of $p<0.05$ were considered statistically significant.

\section{CONFLICTS OF INTEREST}

None declared.

\section{GRANT SUPPORT}

This research was supported by the Intramural Research Program of the National Institute of Health, National Cancer Institute, Center for Cancer Research.

\section{REFERENCES}

1. Frangioni JV. New technologies for human cancer imaging. J Clin Oncol. 2008; 26:4012-4021.

2. Wallace MB, Kiesslich R. Advances in endoscopic imaging of colorectal neoplasia. Gastroenterology. 2010; 138:2140-2150.

3. Weissleder R, Pittet MJ. Imaging in the era of molecular oncology. Nature. 2008; 452:580-589.

4. Keereweer S, Kerrebijn JD, van Driel PB, Xie B, Kaijzel EL, Snoeks TJ, Que I, Hutteman M, van der Vorst JR, Mieog JS, Vahrmeijer AL, van de Velde CJ, Baatenburg de Jong RJ, et al. Optical image-guided surgery-where do we stand? Mol Imaging Biol. 2011; 13:199-207.

5. Achilefu S. Rapid response activatable molecular probes for intraoperative optical image-guided tumor resection. Hepatology. 2012; 56:1170-1173.

6. Kobayashi H, Ogawa M, Alford R, Choyke PL, Urano Y. New strategies for fluorescent probe design in medical diagnostic imaging. Chem Rev. 2010; 110:2620-2640.
7. Kobayashi H, Choyke PL. Target-cancer-cell-specific activatable fluorescence imaging probes: rational design and in vivo applications. Acc Chem Res. 2011; 44:83-90.

8. Razgulin A, Ma N, Rao J. Strategies for in vivo imaging of enzyme activity: an overview and recent advances. Chem Soc Rev. 2011; 40:4186-4216.

9. Urano Y, Sakabe M, Kosaka N, Ogawa M, Mitsunaga M, Asanuma D, Kamiya M, Young MR, Nagano T, Choyke PL, Kobayashi H. Rapid cancer detection by topically spraying a gamma-glutamyltranspeptidase-activated fluorescent probe. Sci Transl Med. 2011; 3:110ra119.

10. Hanigan MH, Frierson HF, Jr., Brown JE, Lovell MA, Taylor PT. Human ovarian tumors express gamma-glutamyl transpeptidase. Cancer Res. 1994; 54:286-290.

11. Yao D, Jiang D, Huang Z, Lu J, Tao Q, Yu Z, Meng X. Abnormal expression of hepatoma specific gamma-glutamyl transferase and alteration of gamma-glutamyl transferase gene methylation status in patients with hepatocellular carcinoma. Cancer. 2000; 88:761-769.

12. Schafer C, Fels C, Brucke M, Holzhausen HJ, Bahn H, Wellman M, Visvikis A, Fischer P, Rainov NG. Gammaglutamyl transferase expression in higher-grade astrocytic glioma. Acta Oncol. 2001; 40:529-535.

13. Hanigan MH, Frierson HF, Jr., Swanson PE, De Young BR. Altered expression of gamma-glutamyl transpeptidase in human tumors. Hum Pathol. 1999; 30:300-305.

14. Ueo H, Shinden $\mathrm{Y}$, Tobo $\mathrm{T}$, Gamachi A, Udo M, Komatsu H, Nambara S, Saito T, Ueda M, Hirata H, Sakimura S, Takano Y, Uchi R, et al. Rapid intraoperative visualization of breast lesions with gamma-glutamyl hydroxymethyl rhodamine green. Sci Rep. 2015; 5:12080.

15. Sato C, Abe S, Saito Y, So Tsuruki E, Takamaru H, Makazu M, Sato Y, Sasaki H, Tanaka H, Ikezawa N, Yamada M, Sakamoto T, Nakajima T, et al. A pilot study of fluorescent imaging of colorectal tumors using a gammaglutamyl-transpeptidase-activatable fluorescent probe. Digestion. 2015; 91:70-76.

16. Mitsunaga M, Kosaka N, Choyke PL, Young MR, Dextras CR, Saud SM, Colburn NH, Sakabe M, Nagano T, Asanuma D, Urano Y, Kobayashi H. Fluorescence endoscopic detection of murine colitis-associated colon cancer by topically applied enzymatically rapid-activatable probe. Gut. 2013; 62:1179-1186.

17. Fujii $\mathrm{T}$, Kamiya $\mathrm{M}$, Urano $\mathrm{Y}$. In vivo imaging of intraperitoneally disseminated tumors in model mice by using activatable fluorescent small-molecular probes for activity of cathepsins. Bioconjug Chem. 2014; 25:1838-1846.

18. Longmire M, Kosaka N, Ogawa M, Choyke PL, Kobayashi $\mathrm{H}$. Multicolor in vivo targeted imaging to guide real-time surgery of HER2-positive micrometastases in a two-tumor coincident model of ovarian cancer. Cancer Sci. 2009; 100:1099-1104.

19. Chen WT, Shih TT, Chen RC, Lo HY, Chou CT, Lee JM, Tu HY. Blood perfusion of vertebral lesions evaluated 
with gadolinium-enhanced dynamic MRI: in comparison with compression fracture and metastasis. J Magn Reson Imaging. 2002; 15:308-314.

20. Zhang WJ, Niven RM, Young SS, Liu YZ, Parker GJ, Naish JH. T1-weighted Dynamic Contrast-enhanced MR Imaging of the Lung in Asthma: Semiquantitative Analysis for the Assessment of Contrast Agent Kinetic Characteristics. Radiology. 2016; 278:906-916.

21. Zhou G, Chen X, Zhang J, Zhu J, Zong G, Wang Z. Contrast-enhanced dynamic and diffusion-weighted MR imaging at 3.0T to assess aggressiveness of bladder cancer. Eur J Radiol. 2014; 83:2013-2018.

22. Okuda S, Oshio K, Shinmoto H, Tanimoto A, Asada H, Fujii T, Yoshimura Y, Kuribayashi S. Semiquantitative assessment of MR imaging in prediction of efficacy of gonadotropin-releasing hormone agonist for volume reduction of uterine leiomyoma: initial experience. Radiology. 2008; 248:917-924.

23. Corti A, Franzini M, Paolicchi A, Pompella A. Gammaglutamyltransferase of cancer cells at the crossroads of tumor progression, drug resistance and drug targeting. Anticancer Res. 2010; 30:1169-1181.

24. Pompella A, De Tata V, Paolicchi A, Zunino F. Expression of gamma-glutamyltransferase in cancer cells and its significance in drug resistance. Biochem Pharmacol. 2006; 71:231-238.

25. Yang M, Luiken G, Baranov E, Hoffman RM. Facile wholebody imaging of internal fluorescent tumors in mice with an LED flashlight. Biotechniques. 2005; 39:170, 172.

26. Hoffman RM, Yang M. Subcellular imaging in the live mouse. Nat Protoc. 2006; 1:775-782.

27. Hoffman RM, Yang M. Color-coded fluorescence imaging of tumor-host interactions. Nat Protoc. 2006; 1:928-935.

28. Hoffman RM, Yang M. Whole-body imaging with fluorescent proteins. Nat Protoc. 2006; 1:1429-1438.

29. Hoffman RM. The multiple uses of fluorescent proteins to visualize cancer in vivo. Nat Rev Cancer. 2005; 5:796-806.

30. Kishimoto H, Zhao M, Hayashi K, Urata Y, Tanaka N, Fujiwara T, Penman S, Hoffman RM. In vivo internal tumor illumination by telomerase-dependent adenoviral GFP for precise surgical navigation. Proc Natl Acad Sci U S A. 2009; 106:14514-14517.

31. Kishimoto H, Urata Y, Tanaka N, Fujiwara T, Hoffman RM. Selective metastatic tumor labeling with green fluorescent protein and killing by systemic administration of telomerase-dependent adenoviruses. Mol Cancer Ther. 2009; 8:3001-3008.

32. Kishimoto H, Aki R, Urata Y, Bouvet M, Momiyama M, Tanaka N, Fujiwara T, Hoffman RM. Tumor-selective, adenoviral-mediated GFP genetic labeling of human cancer in the live mouse reports future recurrence after resection. Cell Cycle. 2011; 10:2737-2741.

33. Yano S, Tazawa H, Hashimoto Y, Shirakawa Y, Kuroda S, Nishizaki M, Kishimoto H, Uno F, Nagasaka T, Urata Y,
Kagawa S, Hoffman RM, Fujiwara T. A genetically engineered oncolytic adenovirus decoys and lethally traps quiescent cancer stem-like cells in $\mathrm{S} / \mathrm{G} 2 / \mathrm{M}$ phases. Clin Cancer Res. 2013; 19:6495-6505.

34. Yano S, Miwa S, Kishimoto H, Uehara F, Tazawa H, Toneri M, Hiroshima Y, Yamamoto M, Urata Y, Kagawa S, Bouvet M, Fujiwara T, Hoffman RM. Targeting tumors with a killerreporter adenovirus for curative fluorescence-guided surgery of soft-tissue sarcoma. Oncotarget. 2015; 6:13133-13148. doi: 10.18632/oncotarget.3811.

35. Yano S, Hiroshima Y, Maawy A, Kishimoto H, Suetsugu A, Miwa S, Toneri M, Yamamoto M, Katz MH, Fleming JB, Urata Y, Tazawa H, Kagawa S, et al. Color-coding cancer and stromal cells with genetic reporters in a patient-derived orthotopic xenograft (PDOX) model of pancreatic cancer enhances fluorescence-guided surgery. Cancer Gene Ther. 2015; 22:344-350.

36. Yano S, Zhang Y, Miwa S, Kishimoto H, Urata Y, Bouvet M, Kagawa S, Fujiwara T, Hoffman RM. Precise navigation surgery of tumours in the lung in mouse models enabled by in situ fluorescence labelling with a killer-reporter adenovirus. BMJ Open Respir Res. 2015; 2:e000096.

37. Yano S, Takehara K, Miwa S, Kishimoto H, Hiroshima Y, Murakami T, Urata Y, Kagawa S, Bouvet M, Fujiwara T, Hoffman RM. Improved Resection and Outcome of ColonCancer Liver Metastasis with Fluorescence-Guided Surgery Using In Situ GFP Labeling with a Telomerase-Dependent Adenovirus in an Orthotopic Mouse Model. PLoS One. 2016; 11:e0148760.

38. Nemunaitis J, Tong AW, Nemunaitis M, Senzer N, Phadke AP, Bedell C, Adams N, Zhang YA, Maples PB, Chen S, Pappen B, Burke J, Ichimaru D, et al. A phase I study of telomerase-specific replication competent oncolytic adenovirus (telomelysin) for various solid tumors. Mol Ther. 2010; 18:429-434.

39. Yano S, Miwa S, Kishimoto H, Toneri M, Hiroshima Y, Yamamoto M, Bouvet M, Urata Y, Tazawa H, Kagawa S, Fujiwara T, Hoffman RM. Experimental Curative Fluorescence-guided Surgery of Highly Invasive Glioblastoma Multiforme Selectively Labeled With a Killer-reporter Adenovirus. Mol Ther. 2015; 23:1182-1188.

40. Hama Y, Urano Y, Koyama Y, Choyke PL, Kobayashi H. D-galactose receptor-targeted in vivo spectral fluorescence imaging of peritoneal metastasis using galactosaminconjugated serum albumin-rhodamine green. J Biomed Opt. 2007; 12:051501.

41. Guide for the Care and Use of Laboratory Animals. Washington, DC: National Academy Press; 1996.

42. Kakareka JW, McCann TE, Kosaka N, Mitsunaga M, Morgan NY, Pohida TJ, Choyke PL, Kobayashi H. A portable fluorescence camera for testing surgical specimens in the operating room: description and early evaluation. Mol Imaging Biol. 2011; 13:862-867. 